\title{
TAGUNG
}

\section{Die polnische EU-Ratspräsidentschaft 2011. Bilanz eines Vorsitzes in Krisenzeiten}

\author{
Susanne Günther*
}

\begin{abstract}
Als Polen am 1. Juli 2011 den Vorsitz im Rat der Europäischen Union übernahm, stellte sich die Frage, ob es einem Nicht-Euro-Staat gelingen würde, die Europäische Union durch die schwierigste Krise seit ihrer Gründung zu führen.
\end{abstract}

Die Rahmenbedingungen für die polnische EU-Ratspräsidentschaft waren alles andere als vielversprechend: Vor allem die europäische Schuldenkrise trieb die Union an den Rand ihrer Handlungsfähigkeit. Die ambitionierten Ziele der polnischen Regierung, die es durch „verstärkte Integration, intensive Zusammenarbeit und vereintes Zusammenwirken"1 umzusetzen gedachte, drohte am bröckelnden europäischen Gemeinschaftsgeist zu scheitern. Zudem musste Polen in seiner Präsidentschaftsrolle auf äußere Ereignisse wie den Arabischen Frühling reagieren. Nationale Prioritäten wie die Wiederbelebung der Östlichen Partnerschaft oder die Vertiefung der EU-Verteidigungspolitik rückten in den Hintergrund.

Letztendlich sollte Polen mit seiner Strategie ,je mehr Europa, desto weniger Krise“2 erfolgreich sein und der polnische Ratsvorsitz aus politischer Sicht der EU-Mitgliedstaaten positiv beurteilt werden. Im Fokus der Tagung „Polen als Motor des europäischen Integrationsprozesses" stand eine bilanzierende Wertung der polnischen EU-Ratspräsidentschaft aus den Perspektiven der Politik-, Rechts- und Wirtschaftswissenschaft.

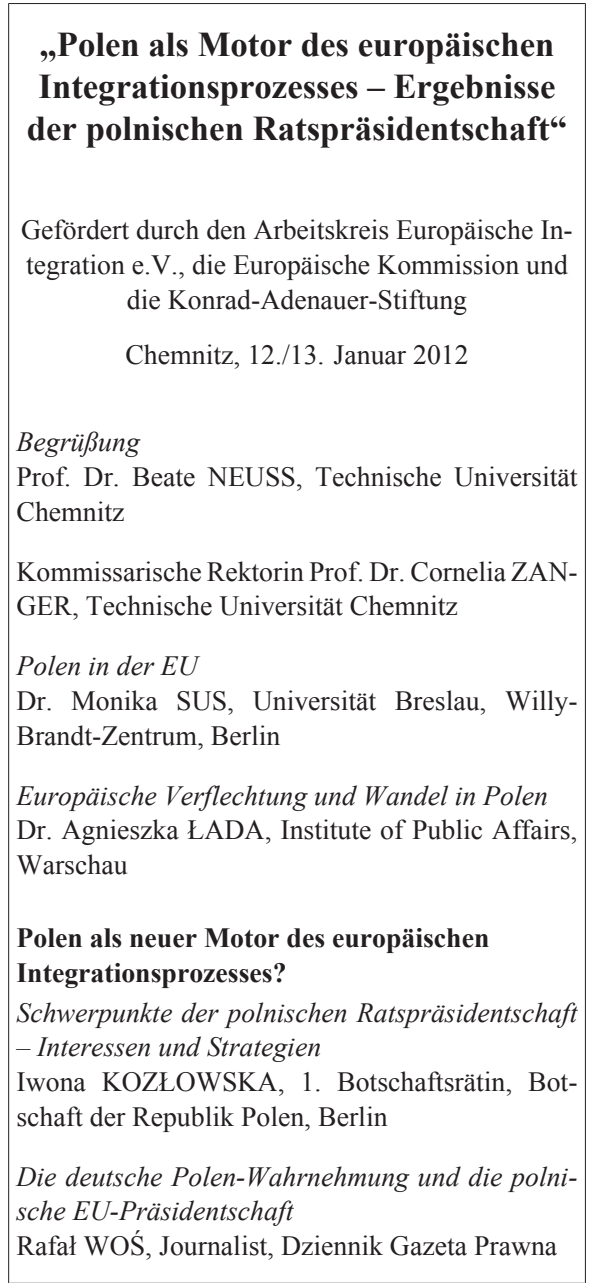

* Susanne Günther, M.A., ist wissenschaftliche Mitarbeiterin der Professur Internationale Politik, Technische Universität, Chemnitz.

1 Vgl. Ministerium für Auswärtige Angelegenheiten: Programm der polnischen EU-Ratspräsidentschaft. 1. Juli bis 31. Dezember 2011, Warschau 2011.

2 Donald Tusk zitiert nach Frankfurter Allgemeine Zeitung: Tusk: Mehr Europa, weniger Krise, 10.7.2011. 


\section{„Von einem Traum zur Realität": Polens Weg in die $E U$}

Im ersten Tagungsbeitrag zeichnete Monika Sus den Weg Polens in die Europäische Union nach. Die Neuorientierung der Außen- sowie der Nachbarschaftspolitik Polens nach dem Ende des Kalten Krieges sei vielschichtig und kompliziert gewesen. Der polnische Staat habe sich an all seinen Grenzen neuen Nachbarn gegenüber gesehen. Die erste freie Parlamentswahl sei der Ausgangspunkt der Westorientierung des polnischen Staates gewesen. Die eingeleitete Transformation habe Polen in eine Art Schockzustand versetzt. Oberste Prioritäten seien die Wiederherstellung rechtsstaatlicher Prinzipien sowie die Überführung des planwirtschaftlich geprägten Wirtschaftssystems in eine soziale Marktwirtschaft gewesen. Die politische Neuorientierung des Staates sei aufgrund der ,innenpolitischen Umbauarbeiten" zunächst zurückgestellt worden. Die Instabilität und Mannigfaltigkeit der politischen Gruppierungen habe die Initialisierung einer konstanten Außenpolitik erschwert.

Sus betonte in ihrem Beitrag, dass der „Weg zurück nach Europa“ nur „über Deutschland“ führen konnte und dass dies in der polnischen Politik allgemein akzeptiert gewesen sei. Die unter polnischen Politikern ebenfalls weit verbreitete Auffassung, dass ,nicht Polen Europa verlassen habe, sondern Europa Polen" habe den polnischen Integrationsprozess in die Europäische Union zunächst gehemmt. Dass Europa um Polen ,werben müsse“, sei bis zur Ratifizierung des Europa-Abkommens 1991 ebenfalls Konsens gewesen.

Die Modernisierung Polens sei durch die 1997 begonnenen Beitrittsverhandlungen mit der Europäischen Union beschleunigt worden. Trotz einiger ungelöster Fragen habe im Beitrittsjahr 2004 ein parteiübergreifender Konsens über die Notwendigkeit der EU-Mitgliedschaft geherrscht. Für Sus stellt diese Entwicklung ein eindeutiges Zeichen des Wandels der Einstellung polnischer Politiker gegenüber der Europäischen Union dar. Das Referendum zur
Bürde der Präsidentschaft - Polens Beitrag zur Konsolidierung des Euro und zur Überwindung der europäischen Schuldenkrise

Dr. Sebastian PŁÓCIENNIK, Universität Breslau, Willy-Brandt-Zentrum, Breslau

Außen-, Sicherheits- und Verteidigungspolitik unter besonderer Berücksichtigung der Beziehungen zu den östlichen Nachbarn

Dr. Kai-Olaf LANG, Stiftung Wissenschaft und Politik, Berlin

Von der polnisch-ukrainischen Grenze nach Schengen. Freizügigkeit, Innen- und Justizpolitische Zusammenarbeit

Prof. Dr. Matthias NIEDOBITEK, Technische Universität Chemnitz

Europäisierung Polens: Gesellschaft und politisches System

Kohäsionspolitik und polnische Regionen

Prof. Dr. Stefan GARSZTECKI, Technische Universität Chemnitz

„Die polnische EU-Ratspräsidentschaft: Evaluations- und Analysekriterien “

Prof. Dr. Ireneusz KAROLEWSKI, Universität Breslau, Willy-Brandt-Zentrum, Breslau

Polen, Deutschland und die Europäische Einigung Prof. Dr. Burkard STEPPACHER, Konrad-Adenauer-Stiftung, Berlin/Universität zu Köln

Zusammenfassung und Schlussbemerkungen

Prof. Dr. Beate NEUSS, Technische Universität, Chemnitz

Ratifikation des Beitrittsvertrags wurde durch die polnische Bevölkerung mit 77,45 Prozent der Stimmen positiv beschieden. Mit der Übernahme der EU-Ratspräsidentschaft sei Polen, so Sus, von einer Konsolidierungs- in eine Emanzipierungsphase eingetreten, in der nicht mehr von „europäischer Polenpolitik“, sondern von „Polens Europapolitik“ gesprochen werden muss.

\section{Polens Gesellschaft als Herausforderung für die Beitrittsverhandlungen}

Agnieszka Łada ging in ihrem Vortrag der Vermutung, dass die polnische Gesellschaft die größte Herausforderung im Rahmen der Bei- 
trittsverhandlungen gewesen sei, empirisch nach und erörterte die europäische Verflechtung und den daraus resultierenden Wandel in Polen. Bei näherer Analyse des Programms des polnischen EU-Ratsvorsitzes ließen sich einige Schlüsselworte filtern - gemeinsam, europäisch, solidarisch, mehr und nicht weniger Europa - die erste Aufschlüsse über die positive Einstellung der Polen zur Europäischen Union geben würden.

Łada argumentierte weiterhin, dass die Polen in der Europäischen Union ,angekommen“ seien und sich als Europäer sowie Unionsbürger identifizieren. Die pro-europäische polnische Politik sei eine logische Konsequenz aus den Einstellungen der polnischen Gesellschaft. Łada merkte jedoch kritisch an, dass nach wie vor zu wenig Wissen über und Engagement für die Europäische Union vorhanden seien, obwohl die Polen den EU-Organen mehr als den nationalen politischen Institutionen vertrauten. Genaue Kenntnisse darüber, wie das Europäische Parlament gewählt wird, existieren beispielswiese nicht.

Łada resümierte, dass die Europafreundlichkeit der Polen sehr stark problemorientiert sei. Wirtschaft, Arbeitslosigkeit und Migration seien die Herausforderungen, die nach Auffassung der Polen durch effektive Maßnahmen der Europäischen Union besser gelöst werden können als auf nationaler Ebene. Daraus resultiere das Institutionen-Vertrauen und die positive Grundhaltung zur Europäischen Union. Łada sieht in der Übernahme des Amtes der Ratspräsidentschaft die Chance für nachhaltiges polnisches Engagement und die Verantwortungsübernahme auf EU-Ebene.

\section{Polen als Impulsgeber für ein modernes Europa}

Iwona Kozłowska stellte in ihrem Beitrag die Interessen und Strategien der polnischen EURatspräsidentschaft dar. Maßgebliche polnische Ziele waren: erstens, die Republik Polen als verlässlichen Partner zu etablieren; zweitens, aktiv das künftige ,politische Gesicht“ der Union mitzugestalten; und drittens, Begeisterung für die Europäische Union - trotz der Krise - zu schaffen.

Kozłowska bezeichnete diese Ziele als äußerst ambitioniert, da die Europäische Union beim Antritt der Ratspräsidentschaft Polens aufgrund der Wirtschafts- und Finanzkrise an einem existenziellen Wendepunkt gestanden habe. Zudem sei eine erfolgreiche Ratspräsidentschaft innenpolitisch von symbolischer Signifikanz, da im Oktober 2011 Parlamentswahlen anstanden. Die Wiederwahl der polnischen Regierung bewertete Kozłowska als ersten Hinweis auf die überzeugende Arbeit der Polen auf europäischer Ebene.

Sie betonte weiterhin, dass die Nichtmitgliedschaft in der Euro-Zone die EU-Ratspräsidentschaft erleichtert habe. Polen habe die Rolle als Mittler zwischen den Euro-Zonen-Staaten und den Nicht-Euro-Staaten übernommen. Der polnische Ratsvorsitz habe eine Fiskalunion initiiert und Pläne für die Teilnahme an wirtschaftlichen Steuerungsprozessen auch für Staaten außerhalb der Euro-Zone vorgelegt. Die Beschlüsse zu einer vertieften Wirtschaftsund Finanzkoordination innerhalb der Europäischen Union ließen sich, so Kozłowska, auf das Verhandlungsgeschick der Polen zurückführen.

Kozłowska betonte, dass es Polen gelungen sei, die Europäische Union auch in der Krise „zu regieren“. Diese Aussage untermauerte sie durch die Aufzählung weiterer polnischer Initiativen und Abkommen, die die Gestalt der Europäischen Union auch nach dem Ende des polnischen Ratsvorsitzes bestimmen werden, wie die Aktivitäten zur Östlichen Partnerschaft und mit Blick auf die südlichen Nachbarn sowie im Bereich der Gemeinsamen Sicherheitsund Verteidigungspolitik (GSVP).

Polen habe während seiner Ratspräsidentschaft bewiesen, dass es Impulsgeber für ein modernes Europa ist. Kozłowska sieht dies auch in den deutsch-polnischen Beziehungen reflektiert, die sich von einer deutsch-polni- 
schen Zusammenarbeit in Europa zu einer Partnerschaft für Europa entwickelt haben.

\section{Der deutsche Blick auf Polen}

Anknüpfend an erste Überlegungen zum Zustand der deutsch-polnischen Beziehungen vor und nach dem Ratsvorsitz Polens, diskutierte Rafat Woś die Polen-Wahrnehmung der Deutschen im Hinblick auf die EU-Ratspräsidentschaft. Zunächst erörterte er die positive Perzeption Polens aufgrund seiner guten Wirtschaftsdaten und der Popularität Donald Tusks. Für Woś markierte der polnische Ratsvorsitz jedoch kein neues Kapitel in den Beziehungen zwischen Deutschland und Polen.

Der Appell des polnischen Außenministers Radosław Sikorski zum Ende der Ratspräsidentschaft, Deutschlands Unterstützung und Führung bei der Überwindung der EU-Schuldenkrise sei nötig, wertete Woś als Ausdruck enttäuschter Hoffnungen Polens. Die EU-Ratspräsidentschaft habe keine Annäherung Polens und Deutschlands zur Folge gehabt, Polen habe eine Neudefinition und Vertiefung der bilateralen Beziehungen im Sinne des ,europäischen Projekts" lediglich fordern können.

\section{Eine Präsidentschaft im Zeichen der Krise}

Sebastian Płóciennik beleuchtete in seinem Vortrag den polnischen Beitrag zur Konsolidierung des Euro und zur Überwindung der europäischen Schuldenkrise. Ausgehend von dem Argument, dass der steigende Einfluss Polens in Europa nicht zuletzt auf die positive wirtschaftliche Entwicklung des Staates zurückzuführen sei, analysierte er zunächst verschiedene Faktoren - Kapital, Arbeit, Qualifikation, Fiskalpolitik - und deren Einfluss auf Stabilität/Instabilität der Wirtschaft.

Der polnische Kapitalmarkt sei klein und offen, die Regulierung und Aufsicht des Marktes funktioniere gut. Der Modernisierungsgrad des Marktes ist aufgrund der geringen Bereitschaft zur privaten Verschuldung gering, der Markt für Finanzdienstleistungen unterentwickelt.
Dennoch, so Płóciennik, sei der reformbedürftige Kapitalmarkt im Vergleich zu den anderen europäischen Märkten stabil geblieben. Polens flexibler Arbeitsmarkt, die diversifizierte Wirtschaftsstruktur sowie der hohe Grad betrieblicher Bildung hätten sich bewährt. Polens niedrige Exportabhängigkeit und die hohe Binnennachfrage hätten dazu beigetragen, dass Polen die Wirtschafts- und Finanzkrise ohne größere Probleme überstand. Polen befinde sich seit 20 Jahren in einem permanenten Transformationsprozess. Die währungspolitische Strategie der polnischen Regierungen sei erfolgreich.

Płóciennik merkte abschließend an, dass es für Polen derzeit keine Option sei, der Euro-Zone beizutreten. Während der polnischen EU-Ratspräsidentschaft habe sich gezeigt, dass die Maastricht-Kriterien einer Reform bedürfen und die Euro-Staaten ihr Währungssystem in Ordnung bringen müssen.

\section{Der Wandel polnischer Außen- und Sicherheitspolitik}

Nach der Beurteilung der polnischen Ratspräsidentschaft aus wirtschaftswissenschaftlicher Perspektive, widmete sich Kai-Olaf Lang der außen-, sicherheits- und verteidigungspolitischen Strategie Polens unter besonderer Berücksichtigung der Beziehungen zu den östlichen Nachbarn.

Polens Ziel sei es gewesen, während seiner Ratspräsidentschaft Akzente im Rahmen der GSVP zu setzen. Die Stärkung der Östlichen Partnerschaft vor dem Hintergrund der ,Versicherheitlichung" polnischer Ostpolitik und der Milderung des Wohlstandsgefälles zwischen EU-Mitgliedstaaten und Nichtmitgliedstaaten seien ein erklärtes Vorhaben gewesen.

Lang erörterte zunächst den Wandel polnischer Außen- und Sicherheitspolitik im europäischen Kontext, der sich in fünf Phasen gliedern lasse:

Die erste Phase sei durch die Neuorientierung der Außen- und Sicherheitspolitik zwischen 
1989 und 1993 gekennzeichnet. Polen habe seine Souveränität zurückgewonnen. Über die künftige Sicherheitsarchitektur Europas habe hohe Unsicherheit geherrscht. Neben der Westorientierung des polnischen Staates hätten sich erstmals Ideen eines sicherheits- und wirtschaftspolitischen Ostverbundes entwickelt. Kontakte $\mathrm{zu}$ den osteuropäischen Nachbarn seien in dieser Phase forciert worden.

In der zweiten Phase von 1993 bis 2004 habe der jagiellonische Konsens gegolten. Die alte Idee einer multiethnischen Konföderation sei an die herrschenden Rahmenbedingungen angepasst worden. Ziel sei die „Westernisierung“ der Mittelstaaten gewesen. Polen habe in dieser Zeit eine antizipierte Rolle in der NATO und in der Europäischen Union zu spielen begonnen. Anrainer wie die Ukraine oder Weißrussland seien vor dem Hintergrund als äußerste Grenze des zivilisierten Europa gegen den ,wilden Osten' betrachtet worden. Im Jahr 1998 sei erstmals die Idee der Östlichen Dimension sowie der Heranführung von sechs osteuropäischen, zum Teil benachbarten Staaten im Rahmen der Europäischen Nachbarschaftspolitik (ENP) entstanden, aber noch nicht verwirklicht worden.

Erst in der dritten Phase ab 2004 sei der jagiellonische Prozess intensiviert und die aktive Arbeit an der „Veröstlichung“ der GSVP begonnen worden. Polen habe die Rolle des Motors und Vermittlers in der Ost-Debatte übernommen, ohne dass die enge transatlantische Bindung infrage gestellt worden sei.

In der vierten Phase, sei eine pragmatische Neuorientierung verbunden mit dem Abschied von der jagiellonischen Idee erst nach den Parlamentswahlen 2007 möglich gewesen. Die Östliche Partnerschaft sei 2008 beschlossen worden. Die Intensivierung der Zusammenarbeit mit Armenien, Aserbaidschan, Georgien, Moldawien, der Ukraine und Weißrussland habe ebenso im Fokus polnischer Ost-Politik wie die Versöhnung mit Russland gestanden.
Der Modernisierungsschub in den Beziehungen Polens zu dessen östlichen Nachbarn sollte in der fünften Phase - dem polnischen EURatsvorsitz - fortgesetzt werden. Die Östliche Partnerschaft sei unter dem Cluster ,[e]in Europa, das von seiner Offenheit profitiert" als Priorität aufgeführt worden. Ziel sei die langfristige wirtschaftliche Integration der östlichen Nachbarn mit deren Einbindung in den Binnenmarkt gewesen.

Lang resümierte, dass es Polen trotz der Limitierungen dennoch gelungen sei, gute Arbeit während des Ratsvorsitzes zu leisten, auch wenn das Ergebnis lediglich moderat sei. Dies führte Lang jedoch auf die zu hohen polnischen Zielsetzungen zurück.

Ferner merkte Lang an, dass Polen im Kontext der östlichen Partnerschaft generell zu passiv agiere. Die Beziehungen seien durch den Anspruch des Wahrens, Sicherns und Rettens charakterisiert. Die polnische Rolle im Rahmen der GSVP sei auch künftig durch Pragmatismus und das Primat staatlicher Sicherheit gekennzeichnet sein.

\section{Erfolge bei der Zusammenarbeit im Raum der Freiheit, der Sicherheit und des Rechts}

An die Ausführungen Langs anknüpfend, widmete sich Matthias Niedobitek der polnischen Nachbarschaftspolitik unter den Aspekten der Freizügigkeit sowie der innen- und justizpolitischen Zusammenarbeit unter Berücksichtigung des polnisch-ukrainischen Grenzraums.

Er führte aus, dass der Beitritt Polens zum Schengen-Abkommen 2007 auf beiden Seiten der polnisch-ukrainischen Grenze eine rege Debatte über das künftige bilaterale Verhältnis ausgelöst habe. Polen habe sich mit zwei Problemen konfrontiert gesehen: erstens die Aufgabe des Schutzes der EU-Außengrenzen und die Kontrolle der Migration in die Europäische Union sowie zweitens die Neudefinition der Beziehungen zu den östlichen Nachbarn unter Berücksichtigung der Zugehörigkeit zum Schengenraum. 
Niedobitek stellte fest, dass es Polen während seiner Ratspräsidentschaft gelungen sei, Erfolge im Bereich der grenzüberschreitenden $\mathrm{Zu}$ sammenarbeit im Raum der Freiheit, der Sicherheit und des Rechts zu erzielen, die den polnisch-ukrainischen Beziehungen zuträglich waren. Auf europäischer Ebene seien die Ergebnisse eher als gering zu bezeichnen, was jedoch auf die schwierigen Rahmenbedingungen der EU-Ratspräsidentschaft sowie die politischen Entwicklungen in der Ukraine zurückzuführen sei. In der anschließenden Diskussion wurde Skepsis hinsichtlich der langfristigen Perspektive von Visaerleichterungen für die Ukraine geäußert. Die Blockadehaltung von Schengenstaaten wie Deutschland sei zu wirkmächtig. Niedobitek wies darauf hin, dass die Fußball-Europameisterschaft im Sommer 2012 Aufschlüsse über die künftige Entwicklung geben könnte, da polnische und ukrainische sowie interne und externe Perspektiven den Raum der Freiheit, der Sicherheit und des Rechts betreffend - das Für und Wider des Umfangs von Grenzkontrollen - sichtbar werden könnten.

\section{Neue Akzente in der Kohäsionspolitik}

Stefan Garsztecki widmete sich in seinem Vortrag der Kohäsionspolitik und deren Bedeutung für die polnischen Regionen. Er setzte Kohäsionspolitik mit Regionalpolitik gleich, da ein großer Teil des jährlichen Budgets in die Förderung der Kooperation von und zwischen Staaten und Regionen fließe.

In Polen sei seit dem Umbruch von 1989 ein zunehmender Prozess der Regionalisierung und Dezentralisierung zu beobachten, der durch nationale und europäische Akteure vorangetrieben werde. Bis 2004 sei die polnische Regionalpolitik durch institutionelle und funktionale Effizienzüberlegungen gekennzeichnet gewesen. Die territoriale Selbstverwaltung sei 1990 umgesetzt worden. Im Rahmen einer Verwaltungsreform seien Kreise (Powiat) wiedereingeführt worden. Seit 2002 würden Bürgermeister und Stadtpräsidenten direkt gewählt, die ausgeglichene Entwicklung der pol- nischen Landesteile werde durch ein Gesetz über die Prinzipien zur Förderung der Regionalentwicklung sichergestellt.

Im Jahr 2004 sei ein Paradigmenwechsel in der polnischen Kohäsionspolitik erkennbar geworden. Die polnischen Regionen agierten immer öfter auf europäischer Ebene. Nach der Parlamentswahl 2007 habe die Regierung von Ministerpräsident Donald Tusk die zentrale Bedeutung der Dezentralisierung betont, um die Bürgergesellschaft weiter zu stärken. Zentrales Vorhaben der Regierung sei ein Gesetz über die Gründung von zwölf Metropolregionen im Umkreis der größten polnischen Städte gewesen, die schlussendlich als Kern regionaler Entwicklung fungieren sollten. Die Übertragung von Kompetenzen an regionale Selbstverwaltungskörperschaften habe sich als schwierig erwiesen, da die Vertreter von Regionen und Kommunen auch zusätzliche Kosten befürchteten. Bisher konnte das Metropolgesetz nicht realisiert werden.

Die polnische Regierung bemühe sich um eine bessere Koordination der strategischen Planung der Regionalentwicklung. Zwar habe sich Polen in den vergangenen Jahren sozioökonomisch dem EU-Durchschnitt angenähert, aber die regionalen Disparitäten zwischen den Subregionen wüchsen. Auf der Grundlage der Prinzipien der Nicht-Diskriminierung, der Solidarität sowie der territorialen Kohäsion seien neun Strategien formuliert worden, die zur Angleichung der regionalen Entwicklung beitragen sollten. Sie umfassten unter anderem einen effizienten Staat, effiziente Wirtschaft, und nationale Sicherheit. Diese sollten auf dem nationalen Entwicklungsplan „Bericht Polen 2030" aufbauen.

Polen habe während seines Ratsvorsitzes die Ausrichtung der Kohäsionspolitik an allen Regionen der Europäischen Union akzentuiert. Es habe das Konzept der Schaffung eines Gemeinsamen Strategischen Rahmens für die verschiedenen Politiken forciert, unter anderem Agrar- und Fischereipolitik. Partnerschaftliche Entwicklungs- und Investitionsverträge sollten 
deren Integration erleichtern. Die polnische Regierung plädierte während der EU-Ratspräsidentschaft zudem für eine Stärkung der territorialen Dimension der Kohäsionspolitik und habe sich für eine Förderung der Regionen auf allen Verwaltungsebenen stark gemacht.

Garsztecki stellte abschließend fest, dass die europäische Kohäsionspolitik die treibende Kraft der Entwicklung der Regionalpolitik Polens darstelle. Das Wirtschafts- und Innovationspotenzial der polnischen Republik sei durch die umfassende Dezentralisierung erhöht worden. Im Rahmen der Ratspräsidentschaft sei es Polen gelungen, die Verhandlungen über die zukünftige Gestalt der Kohäsionspolitik voranzutreiben. Die öffentliche Wahrnehmung der Inhalte von Kohäsionspolitik sei jedoch gering.

\section{Ein Kriterien-Raster zur Analyse von Ratspräsidentschaften}

Ireneusz Karolewski wandte sich der polnischen EU-Ratspräsidentschaft aus sozialwissenschaftlicher Perspektive zu. Er präsentierte Evaluations- und Analysekriterien zur qualitativen und quantitativen Bewertung von Ratspräsidentschaften. Die gegenwärtige Forschung beruhe auf Einzelfallstudien und weise wenig Systematik auf. Durch die „Triopräsidentschaft" und dem Wechsel des Vorsitzes im Sechsmonatszyklus seien die EU-Ratspräsidentschaften in ihrer Relevanz gesunken. Interessanter erscheine die Analyse politischer, wirtschaftlicher und gesellschaftlicher Impulsgeber für die Präsidentschaftsprogramme sowie die ,leadership capacity ${ }^{\circ}$ der jeweiligen Präsidentschaft.

Die methodische Annäherung zur Schaffung eines Analyserasters bezeichnete Karolewski aufgrund der hohen Ambivalenz der Kriterien als schwierig. Eine Untersuchung könne zunächst auf der funktionalen Identifikation rechtlich-formaler, politischer sowie subjektiver Kriterien fußen. Zur Evaluation einer Ratspräsidentschaft empfehlen sich drei Beobachtungsebenen: erstens, externe Erwartungen der
Partner; zweitens, interne Erwartungen im eigenen Staat; und drittens, Kontingenzen interner und externer Einflüsse.

Hinsichtlich der Bewertung von Ratspräsidentschaften sollten diametrale Strategien der Amtsausübung berücksichtigt werden: erstens die Strategie des ,honest broker' und zweitens die Strategie des ,prudent president'. Während das Präsidentschaftsprogramm des, honest broker' europäische Interessen und die Umsetzung langfristiger Ziele unter Einbeziehung aller EU-Institutionen betont, ist das Programm des ,prudent president ${ }^{\star}$ durch nationale Interessen und einen engen Aktionsrahmen gekennzeichnet. Im Hinblick auf die polnische Ratspräsidentschaft lasse sich, so Karolewski, eine klare Tendenz zum ,honest broker' feststellen. Ein weiteres Merkmal der ,honest broker'Strategie sah Karolewski in der Vertiefung der Koordination zwischen EU-Mitgliedern und Drittstaaten sowie Euro-Zonen-Staaten und Nicht-Euro-Zonen-Staaten.

Vorbehaltlich weiterer Analysen zog Karolewski dennoch eine erste positive Bilanz der polnischen Ratspräsidentschaft. Es sei Polen gelungen, trotz der Krise, politische, gesellschaftliche und mediale Akzente zu setzen.

Etappen auf dem Weg zu einer europäischen Partnerschaft: Deutschland und Polen

Burkard Steppacher widmete sich in seinem Beitrag der deutsch-polnischen Geschichte und zeichnete die wichtigsten historischen Ereignisse auf dem Weg zur Partnerschaft im Rahmen der Europäischen Union nach. Er erinnerte anhand von Karikaturen, Bildern und historischen Dokumenten an Krisen-, Wendeund Schlüsseljahre der deutsch-polnischen Beziehungen.

Zunächst wurde angemerkt, dass die Darstellung der tausendjährigen Beziehungsgeschichte der Deutschen und Polen ein schwieriges Unterfangen sei. Das nachbarschaftliche Verhältnis sei über Jahrhunderte unterschiedlich ausgeprägt gewesen. Auf Perioden friedlichen 
Miteinanders folgten destruktive Phasen der Unversöhnlichkeit. Das Hin und Her zwischen Annäherung und Ablehnung sei ein charakteristisches Merkmal der deutsch-polnischen Nachbarschaft.

Als Krisenjahre bezeichnete Steppacher die Nachkriegsära, in der die Frage der Anerkennung der Oder-Neiße-Linie als deutsch-polnische Staatsgrenze zentral gewesen sei. Die Unterzeichnung des Görlitzer Abkommens 1950 durch Vertreter der Deutschen Demokratischen Republik und Polens markiere ein Schlüsseljahr im bilateralen Verhältnis.

Wendejahre hätten die 1970er Jahre dargestellt: Der 1972 geschlossene „Vertrag zwischen der Bundesrepublik Deutschland und der Volksrepublik Polen über die Grundlagen der Normalisierung ihrer gegenseitigen Beziehungen" (Warschauer Vertrag) umfasste die Anerkennung der Oder-Neiße-Linie als westliche Staatsgrenze Polens.

Die symbolische Kraft des Kniefalls von Willy Brandt vor dem Denkmal der Aufständischen des Warschauer Ghettos im Jahr 1970 habe den Erfolg der vertraglichen Vereinbarung um ein vielfaches überstrahlt.

Die deutsch-polnischen Beziehungen hätten seit dem Aufleben demokratischer Transformationsprozesse in den Jahren 1989/90 maßgeblichen Veränderungen unterlegen. Dem innenpolitischen Wandel sei die endgültige Lösung der deutsch-polnischen Grenzfrage im Rahmen des Zwei-plus-Vier-Vertrages im Jahr 1990 gefolgt.

Diese Entwicklungen hätten den Weg für einen beziehungsgeschichtlichen Kurswechsel geebnet. In Hinblick auf die wirtschaftliche und politische Kooperation im Rahmen der Europäischen Union sei dieser Wechsel geglückt, so Steppacher. Der polnische NATO-Beitritt 1999, der EU-Beitritt 2004 sowie die Übernahme der EU-Ratspräsidentschaft hätten die Errichtung einer auf Freundschaft und Zusammenarbeit basierenden Partner- und Interessengemeinschaft flankiert.

\section{Eine positive Bilanz trotzt erheblicher Hürden}

Am Ende der Tagung blieb die Beantwortung der Fragen: Ist es Polen gelungen, die Europäische Union durch die schwierigste Krise seit ihrer Gründung zu führen? Konnte Polen als Motor des europäischen Integrationsprozesses fungieren? Beide Fragen wurden bejaht.

Polen präsentierte sich als ein Staat mit ausgeprägtem europäischem Gemeinschaftsgeist, dem es während seines Ratsvorsitzes gelang, der Europäischen Union neue Impulse zu geben. In der Zeit seiner Präsidentschaft fungierte Polen als Verwalter, Vermittler und Koordinator zwischen den Mitgliedstaaten.

Die polnische Ratspräsidentschaft ist detailliert vorbereit und professionell durchgeführt worden. Aufgrund der Eurokrise und den aus dem Vertrag von Lissabon resultierenden Kompetenzverlusten blieb der Output der polnischen Ratspräsidentschaft gering. Vor dem Hintergrund der Restriktionen sind die polnischen Initiativen jedoch eindeutig positiv zu bewerten.

Polen verdeutlichte durch seine Bemühungen, dass Krisen nur europäisch zu bewältigen sind. Deutlich wurde im Verlauf der Tagung der Reformbedarf in den einzelnen Politikfeldern, um globalen Herausforderungen wie der Finanzund Schuldenkrise gewachsen zu sein. Polnische Initiativen auf europäischer Ebene stellen Arbeitsgrundlagen für künftige EU-Ratspräsidentschaften dar. 